INTERNATIONAL JOURNAL OF SYSTEMATIC BACTERIOLOGY
Vol. 16, No. 2
April. 1966
pp. $191-193$

\title{
CRITIQUE OF MOLLARET'S PROPOSALS CONCERNING THE NAMES PASTEURELLA PSEUDOTUBERCULOSIS AND $\underline{P}$. PESTIS \\ Herbert Haupt
}

\author{
Faculty of Veterinary Medicine \\ University of Giessen \\ Giessen, Germany
}

Mollaret (1864) insists on the double "necsssity":

1. To withdraw from the genus Pasteurella the species Pasteurella pseudotuberculosis (Eisenberg 1891)

Topley and Wilson 1931, and

Pasteurella pestis (Lehmann et Neumann 1896)

Holland 1920 (bacille de Mallassez et Vignal and bacille de Yersin)

2. To reject the epithet pseudotuberculosis.

The International Code of Nomenclature of Bacteria and Viruses (1958) emended (this Bull. 13, No. 1) contains the principles, rules and recommendations, by the recognition of which "the progress of Bacteriology can be furthered."

(1) Transferences of a species to another genus have been performed many times (especially after realizing that the differentiation of species and genera upon other than morphological differences may be wholly valid). The varied definition of a species as "basic category" may necessitate alterations of higher categories of consecutive ranks in ascending sequence (Principle 7a); "the definition of each of these categories varies up to a certain point according to individual opinion and the state of the science" (Principle 7e). Such definitions concern only the discretion and responsibility of the author proposing them and the state of science.

In Rule $18 \mathrm{~b}$ the transference of species to another genus re the fate of the specific epithet is mentioned (see below). Against Mollaret's proposal(1) there are no objections to be derived from the "Code."

(2) In contrast to the generic name the specific epithet of a species name enjoys a special support against alterations. The Code reads: 
a. In Principle 9. ... In species the correct name is binary...combination of the generic name with the earliest available epithet validly published with the same rank."

b. In "Rule 6.' A specific epithet may be taken from any source whatever and may even be arbitrarily composed. Within the same genus no two species names may bear one specific epithet..."

A specific epithet consisting of two or more words, when originally published, is not to be rejected, but when used the words are to be hyphenated or joined. (note to Rule 6)

c. In "Rule $18 \mathrm{~b}$. When a species is transferred to another genus without change of rank, the specific epithet must be retained..."

d. In "Rule 23. A legitimate name or epithet must not be rejected merely because it is inappropriate, or disagreeable or..."

In order to define an attitude to Mollaret's argumentation, the names hitherto given to be nomina ambigua or nomina dubia or nomina confusa (Rule $24 \mathrm{e}-\mathrm{g}$ ) the following list may be given.

a. The chronological sequence of validly published names with the specific epithet pseudotuberculosis is:

Bacillus pseudotuberculosis Eisenberg 1891

Bacterium pseudotuberculosis Migula 1900

Corynebacterium pseudotuberculosis Bergey et al. 1925

Pasteurella pseudotuberculosis Topley et Wilson 1931

Shigella pseudotuberculosis Haupt 1935

Cillopasteurella pseudotuberculosis Haupt 1964

b. With the specific epithet pseudotuberculosis-rodentium ${ }^{1}$

Streptobacillus pseudotuberculosis-rodentium Preib 1894

Bacterium pseudotuberculosis-rodentium Lehmann et Neumann 1896

See above Rule 6. 
Malleomyces pseudotuberculosis-rodentium Pribram 1933 Corynebacterium pseudotuberculosis-rodentium Kelser 1933

Cillopasteurella pseudotuberculosis-rodentium Prévot 1948

c. With the specific epithet rodentium:

Corynebacterium rodentium Bergey et al. 1923

Yersinia rodentium van Loghem 1944

All names combined with a generic name defined by cellwalls of the Gram-positive type with any of the specific epithets characterised by cell-walls of the Gram-negative type are apparent misnomers. As they were formed in the interval between the acknowledgement of biological characteristics besides morphological ones. These errors are intelligible and excusable. They have sunk in oblivion about 40 years ago. All other names with the epithet pseudotuberculosis in combination with a generic name characterised by cell-walls of the Gram-negative type never have been the cause of ambiguity or uncertainty or confusion.

There is only one epithet, "pseudotuberculosis," legitimate!

\section{APPENDIX}

The argumentation, that the designation "pseudotubercular" has been incorrectly applied to the macroscopic lesions produced by many species of bacteria does not at all touch the nomenclature (Rule 6, see above). 
